\title{
Measurement of radiation-induced autophagic flux in human brain cancer cells
}

\author{
Linda S. Yasui ${ }^{1}$, Megan Johnson ${ }^{1}$, Hannah Savage ${ }^{1}$, Abraham Baker ${ }^{1}$, Elle Baker ${ }^{1}$, Colton Burns ${ }^{1}$ \\ 1. Northern Illinois University, Department of Biological Sciences, DeKalb, IL, USA
}

Radiation-induced autophagy mediates susceptibility to radiation [1]. However, autophagy is a multifaceted process. Herein, 2 aspects of autophagy, induction of autophagy or changes in autophagic flux, in radiation sensitivity were investigated. Relying on a systems-based approach and adding to a large, data dense morphome database [2, 3], we mined for complex cellular information on autophagy due to irradiation of human brain cancer (glioblastoma or GBM) cells. A quantitative stereology method is described here to chronicle these two important aspects of autophagy. These results add to our understanding of the role of autophagy in the efficacy of radiation therapy by providing a basis for the adaptive role for radiation-induced autophagy in GBM cells. Further, because GBM tumors are so resistant to radiotherapy, a novel strategy to radiosensitize GBM cells is urgently needed.

Autophagy is a normal, basal housekeeping process in a cell. The main function of autophagy is to recycle damaged or old cellular constituents by encapsulating cargo within autophagosomes that are then trafficked to fuse with lysosomes, allowing lysosomal hydrolytic enzymes to catabolize the cellular material into simple macromolecular subunits. Induction of autophagy above basal levels provided some of the first insights into the complex role of this dynamic process in cell death and its role in radiation sensitization $[4,5]$.

To provide a proof-of-principle, morphomics data was collected and analyzed for human glioblastoma (U87) monolayer cultured cells irradiated with 0 or 10 Gy $\gamma$ rays [2] and 3 days after treatment were collected and fixed for TEM (the most sensitive method to monitor autophagy [6]). Biased sampling methods were used to selectively acquire at least 30 cell profiles for each treatment. The number of autophagosomes was scored for each cell to reflect changes in induction of autophagy in the cell population and within each cell. Autophagic flux was estimated by measuring the area of the cytoplasm occupied by autophagosomes using ImageJ. The area measurement integrates both the number of autophagosomes and size of autophagosomes in the cell. Increased flux, a possible outcome of the area analysis, is revealed when a decreased number and area of autophagosomes is found because hydrolytic digestion of autophagosomes indicates completion of the autophagic process. In contrast, decreased autophagic flux is revealed by increased number or area of autophagosomes resulting from a blockade in autophagy before the final hydrolysis of autophagosomes. Finally, a third outcome of the analysis is the identification of cells that have cytoplasmic areas occupied by autophagosomes that are more than 1 standard deviation of the average area. These outliers focus our attention on those cells having the most dramatic change in radiation-induced autophagy where flux is decreased AND continued autophagosome formation is abnormal. Indeed, previous experiments identified a significant proportion of an irradiated population as outliers, having extremely large autophagosomes due to irradiation with 10 Gy $\gamma$ rays or 2 Gy gadolinium neutron capture [2].

Image analysis results are shown in Fig 1. Fig. 1a shows 10 Gy $\gamma$ irradiation induced autophagy in essentially $100 \%$ of the U87 cells ( $92 \%+/-9 \%)$ compared to only $50 \%+/-18 \%$ of the untreated control cells. Induction of autophagy by irradiation is further supported by our finding of a greater average number of autophagosomes per cell in the $10 \mathrm{~Gy}$ irradiated sample $(2.48+/-1.33)$ compared to 0.73 +/- 
0.83 for unirradiated cells (Fig. 1b). Area measurements exposed a significant decrease in radiationinduced autophagic flux with the finding of a significantly higher area for irradiated cells $(0.59 \%+/-$ $0.86 \%)$ compared to the untreated cells $(0.19 \%+/-0.25 \%)$. The radiation-induced increase in average area certainly reflects the increased number of autophagosomes that are not degraded in the irradiated cells. However, a very large increase in area (21.67\%) was identified by our criteria for an outlier from the 10 Gy irradiated U87 cell sample. In this outlier cell, the increased area resulted from measuring 5 extremely large autophagosome in the image. Although 5 autophagosomes is slightly greater than 1 standard deviation of the mean number of autophagosomes found in the irradiated cells, the large size of the autophagosomes (21.67\% area of the cytoplasm versus $0.59 \%+/-0.86 \%$ ) clearly plays a more significant part in the extremely large area value. Combined with our previous observation of extraordinarily large, radiation-induced autophagosomes [2], these new results support our previous speculation that $10 \mathrm{~Gy}$ irradiation alters a final step for autophagy by promoting continued collection of more and more cargo into the autophagosome, resulting to extremely large autophagosomes.

Quantitative examination of the image database reveals an increase in induction of autophagy by 10 Gy $\gamma$ irradiation with a corresponding decrease in completion of autophagy (decreased flux). Radiationinduced autophagy results in unique, extremely large autophagosomes. Further support for radiationinduced aberrant cargo collection during the final stages of autophagy is pending assessment of more data collected at longer time points after irradiation.

\section{References:}

[1] JM Lucocq et al. Trends in Cell Biology 25 (2015) p. 59-64.

[2] LS Yasui, K Owens. International Journal of Radiation Biology 88 (2012) p. 980-990.

[3] CE Zois, MI Koukourakis. Autophagy 5 (2009) p. 442-450.

[4] K Sharma et al. EXCLI Journal 13 (2014) p. 178-191.

[5] N Mizushima, T. Yoshimori, B. Levine. Cell 140 (2010) p. 313-326

[6] SK Backues et al. Autophagy 10 (2014) p. 155-160.

[7] The authors acknowledge support from a Department of Defense grant W81XH-10-1-017

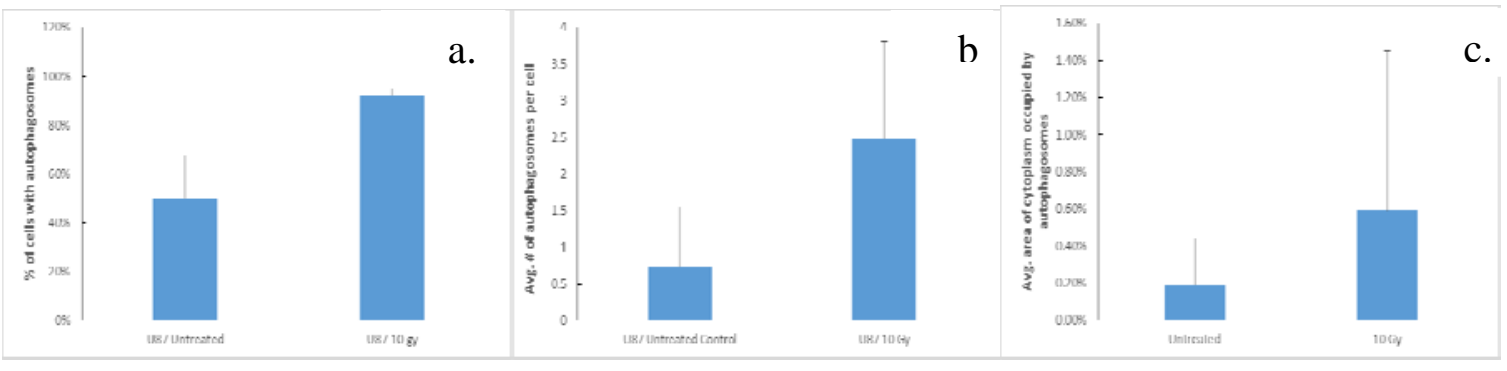

Figure 1. Morphomics of autophagy in U87 GBM cells. (a) Percentage of the population of 0 or 10 Gy irradiated cells that contained autophagosomes +/- 95\% confidence intervals. (b) The average number of autophagosomes per cell profile +/- SD and (c) the average percent area of the cytoplasm occupied by autophagosomes +/- SD. ANOVA analysis, excluding outliers, showed the untreated cell populations were different from the $10 \mathrm{~Gy}$ irradiated population. Post hoc Tukey tests indicated that the untreated control were significantly different from the $10 \mathrm{~Gy}$ irradiated sample $($ at $\mathrm{p}=0.05)$ for graphs (a) and (b). 